\title{
miR-873 acts as a novel sensitizer of glioma cells to cisplatin by targeting Bcl-2
}

\author{
XIONG CHEN $^{1}$, YINGYING ZHANG ${ }^{1}$, YINGYING SHI ${ }^{1}$, HAIWEI LIAN ${ }^{2}$, HUILIN TU $^{1}$, \\ SONG HAN ${ }^{1}$, BIWEN PENG ${ }^{1,3}$, WANHONG LIU ${ }^{1,4}$ and XIAOHUA HE ${ }^{1,3}$ \\ ${ }^{1}$ School of Basic Medical Sciences, Wuhan University; ${ }^{2}$ Department of Neurosurgery, \\ Wuhan University Renmin Hospital; ${ }^{3}$ Hubei Provincial Key Laboratory of Developmentally Originated Disease; \\ ${ }^{4}$ Hubei Province Key Laboratory of Allergy and Immunology, Wuhan 430071, P.R. China
}

Received June 30, 2015; Accepted August 10, 2015

DOI: $10.3892 /$ ijo.2015.3143

\begin{abstract}
Treatment with cisplatin, a chemotherapeutic agent commonly used in glioma patients, often results in chemoresistance. Increasing evidence has shown that microRNAs (miRNAs) are implicated in the drug resistance of gliomas. However, the function of miR-873 in cisplatin resistance of gliomas remains unknown. In this study, we found that many miRNAs, including miR-873, are differentially expressed in cisplatin-resistant glioma cells compared to wild-type glioma cells. Moreover, cisplatin reduced the expression of miR-873 in a time-dependent manner. Overexpression of miR-873 decreased the cell proliferation, migration and invasion while increased apoptosis of cisplatin-resistant glioma cells and sensitized the cells to cisplatin-induced cell growth arrest and apoptosis. Furthermore, miR-873 was downregulated while Bcl-2 was upregulated in the tissues of twelve high-grade glioma patients compared to seven normal brain tissues, and the miR-873 level was negatively correlated with the Bcl-2 protein level. A luciferase reporter assay further confirmed that Bcl-2 was a direct target of miR-873, and miR-873 decreased the level of the Bcl-2 protein in cisplatin-resistant glioma cells. Notably, re-expression of $\mathrm{Bcl}-2$ attenuated the function of miR-873 in cisplatin-resistant glioma cells and the sensitivity of the cells to cisplatin. Taken together, these data suggest that miR-873 might be a potential marker for cisplatin resistance and a promising sensitizer in cisplatin treatment.
\end{abstract}

\section{Introduction}

Gliomas represent the most common primary brain tumors in adults (1). The average survival time for high-grade glioma patients is $\sim 14$ months (2). Even with successful surgical

Correspondence to: Dr Wanhong Liu or Dr Xiaohua He, School of Basic Medical Sciences, Wuhan University, 185 Donghu Road, Wuchang, Wuhan 430071, P.R. China

E-mail: liuwanhong@whu.edu.cn

E-mail: hexiaohua@whu.edu.cn

Key words: miR-873, Bcl-2, cisplatin, glioma resecstion, the prognosis for a patient whose tumor is not sensitive to chemotherapeutic drugs is poor. Cisplatin is well established for use as a chemotherapeutic drug in gliomas (3). However, the molecular mechanisms underlying the chemoresistance of gliomas are largely unclear.

MicroRNAs (miRNAs) are a class of small endogenous noncoding RNAs of 18-25 nucleotides that negatively regulate their target genes by interacting with the 3 '-untranslated region (3'UTR) of the mRNAs (4). The differential expression of miRNA between cancers and their normal counterparts have been explored in various cancers, including glioma (5-9), and these results suggest critical roles for miRNAs in the pathogenesis of the cancers. Recently, accumulating evidence has indicated that miRNAs are implicated in the chemoresistance of gliomas. Let-7b was found to be significantly downregulated in cisplatin-resistant U251 cells compared to wild-type cells and has the ability to improve the therapeutic effect of cisplatin in glioma cells (10). Knockdown of miR-106a enhanced the temozolomide-induced apoptosis in glioma cells (11). Overexpression of miR-136 sensitizes glioma cells to cisplatin by targeting E2F1 and Bcl-2 $(12,13)$. To date, only a few studies have reported the function of miR-873 (14-16). In particular, the effect of miR-873 on the chemoresistance of glioma cells has not been reported.

In this study, we found that hsa-miR-873-5p (miR-873) was downregulated in cisplatin-resistant glioma cells compared to wild-type cells. Furthermore, exposing wild-type cells to cisplatin decreased their expression of miR-873. Further experiments demonstrated that miR-873 decreased the activity of cisplatin-resistant glioma cells and sensitized cisplatinresistant glioma cells to cisplatin by directly targeting Bcl-2, an anti-apoptotic protein expressed in a wide variety of cancers. Ectopic expression of Bcl-2 in cisplatin-resistant glioma cells could prevent the cisplatin-sensitizing effect of miR-873. These results indicated that miR-873 might be a promising therapeutic strategy to overcome cisplatin resistance in gliomas.

\section{Materials and methods}

Patients and samples. Twelve high-grade glioma tissues and seven normal brain tissues were collected between 2011 and 
2014 from the Department of Neurosurgery, Renmin Hospital of Wuhan University. The samples were obtained at the time of surgery and were immediately snap-frozen in liquid until use. The project was approved by the ethics committee of Renmin Hospital.

Cell lines and cell culture. The human glioma cell lines U87 and U251 were purchased from American Type Culture Collection (ATCC). The cells were cultured in Dulbecco's modified Eagle's medium (DMEM, Hyclone, Logan, UT, USA) containing $10 \%$ fetal bovine serum (FBS, Gibco, Grand Island, $\mathrm{NY}, \mathrm{USA})$ at $37^{\circ} \mathrm{C}$ in a $5 \% \mathrm{CO}_{2}$ humidified incubator.

The cisplatin-resistant U87 (U87/DDP) and U251 (U251/ DDP) cell lines were established by prolonged exposure to increasing concentrations of cisplatin (Sigma-Aldrich, St. Louis, MO, USA). The cisplatin concentrations were increased stepwise from 0.1 to $10 \mu \mathrm{g} / \mathrm{ml}$ when the cells resumed growth similar to the untreated cells. U87 cells with the ability to grow in $5 \mu \mathrm{g} / \mathrm{ml}$ of cisplatin and U251 cells with the ability to grow in $0.5 \mu \mathrm{g} / \mathrm{ml}$ of cisplatin were obtained 4 months after the initial drug exposure and were designated U87/DDP and U251/DDP, respectively. The MTT assay results demonstrated that the drug sensitivity of these cells was changed.

RNA extraction and $q P C R$. Total RNA was extracted from human tissues using TRIzol reagent (Invitrogen, San Diego, CA, USA). Reverse transcription was performed using RevertAid First Strand cDNA Synthesis kit (Thermo Scientific, Waltham, MA, USA). To detect miR-873, the Bulge-Loop ${ }^{\text {TM }}$ miRNA qRT-PCR Primer kits (Ribobio, Guangzhou, China) were utilized following the manufacturer's instructions, and qPCR was performed using SuperReal PreMix (SYBR Green) (Tiangen, Beijing, China) with an iCycler thermal cycler (Bio-Rad, Hercules, CA, USA). The relative expression levels were calculated using the $2^{-\Delta \Delta \mathrm{Ct}}$ method after normalization to the expression of U6 snRNA.

MTT assay. The cells were seeded into 96-well plates $\left(3 \times 10^{3}\right.$ cells/well) and incubated for $24 \mathrm{~h}$. The cells were transfected with vectors for another $24 \mathrm{~h}$ and then treated with various concentrations of cisplatin. At 24,48 and $72 \mathrm{~h}$, the cell viability was determined using the MTT solution $(0.5 \mathrm{mg} / \mathrm{ml})$ (SigmaAldrich). After $4 \mathrm{~h}$ of incubation, the medium was discarded and $150 \mu 1$ of dimethyl sulfoxide (DMSO) (Sigma-Aldrich) was added to each well. The plate was vortexed for $30 \mathrm{~min}$, and the absorbance at $570 \mathrm{nM}$ was read using a spectrophotometer. Each experiment was performed in triplicate.

Colony formation assay. The cells transfected with the vectors were trypsinized to single cell suspensions and were seeded into 6-well plates at 1,000/well. Then, after 2 weeks of culture in DMEM with $10 \%$ FBS, the cells were fixed with $4 \%$ paraformaldehyde and stained with $0.5 \%$ crystal violet solution. Each experiment was performed in triplicate.

Luciferase reporter assay. The 3'UTR sequence of Bcl-2 that was predicted to interact with miR-873 was amplified and cloned into the SpeI and HindIII sites of the pMiR-Reporter vector (Ambion, USA). Site-directed mutagenesis of the miR-873 target site was performed using Quik-Change ${ }^{\text {TM }}$
Site-Directed Mutagenesis kit (Stratagene, USA). The constructs were sequenced and named pBcl2-3'UTR-wt or pBcl2-3'UTR-mut. For the reporter assays, the U87/DDP cells were seeded into 24-well plates and transfected with either $50 \mathrm{ng}$ of pBcl2-3'UTR-wt or pBcl2-3'UTR-mut and the miR-873 or NC mimics (RiboBio) with Lipofectamine 2000 (Invitrogen). After incubation for $24 \mathrm{~h}$, cells were harvested and assayed with the Dual-Luciferase Reporter Assay kit (Promega, Wisconsin, MA, USA) according to the manufacturer's instructions.

Cell migration and invasion assays. The detailed methods of cell migration and invasion assays were as described previously (12). Cells $\left(4 \times 10^{4}\right)$ were seeded in the chamber (Corning, NY, USA) for migration assay or invasion assay. The cell number of migration rate and invasion were calculated by photographing at five random fields.

Cell apoptosis assay. After being transfected with the vectors for $24 \mathrm{~h}$, the cells were treated with cisplatin for another $48 \mathrm{~h}$, then detached by trypsinization, centrifuged, washed in phosphate-buffered saline(PBS), resuspended in Annexin V-binding buffer and incubated with Annexin V-phycoerythrin (PE) and 7-amino-Actinomycin D (7AAD) (KeyGen Biotech, Nanjin, China) for $15 \mathrm{~min}$. The rate of apoptosis was determined using flow cytometry.

Caspase-3/7 activity analysis. Caspase-3/7 activity was measured using the Caspase-Glo3 assay kit (Promega) according to the manufacturer's instructions.

Western blot assay. The cells were lysed using the mammalian protein extraction reagent RIPA (Beyotime, Beijing, China). Approximately $100 \mu \mathrm{g}$ of the protein sample was separated on a 12\% SDS-PAGE gel and transferred onto a PVDF membrane (Sigma-Aldrich). The membrane was blocked with 5\% skim milk for at least $2 \mathrm{~h}$ at room temperature. Monoclonal antibodies against Bcl-2 (1:1,000, Cell Signaling, Danvers, MA, USA), PARP (1:3,000, Cell Signaling), and $\beta$-actin (1:5,000, Sigma-Aldrich) were added in 3\% BSA and incubated at $4^{\circ} \mathrm{C}$ overnight. The membrane was washed and then incubated with horseradish peroxidase (HRP)-labeled secondary antibody (1:1,000, Sigma-Aldrich) at room temperature for $1.5 \mathrm{~h}$. Then, the membrane was washed, and the proteins were visualized using an ECL kit (Millipore, Billerica, MA, USA).

Statistical analysis. The data are shown as the means \pm SD (standard deviation) and were analyzed using SPSS 17.0 software. Statistical significance was determined using Student's $\mathrm{t}$-test, and $\mathrm{P}<0.05$ was considered to be significantly different. The Spearman rank correlation coefficients were calculated to determine the bivariate correlations between study variables.

\section{Results}

miR-873 was downregulated in glioma tissues and cisplatinresistant glioma cells. Cluster analysis has revealed that several miRNAs including miR-873 are differentially expressed in glioma tissues (17). Therefore, we compared the expression of miR-873 in the tissues from twelve high-grade glioma patients 
A

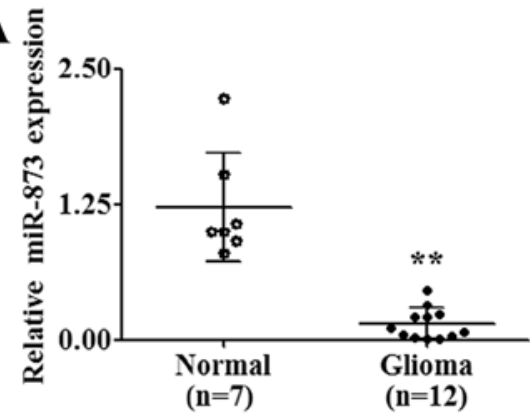

D

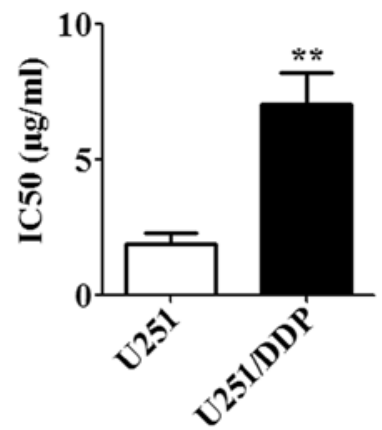

B

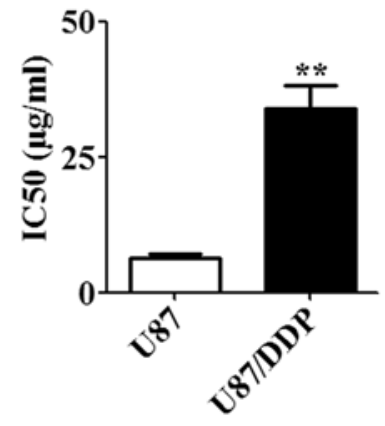

$\mathbf{E}$

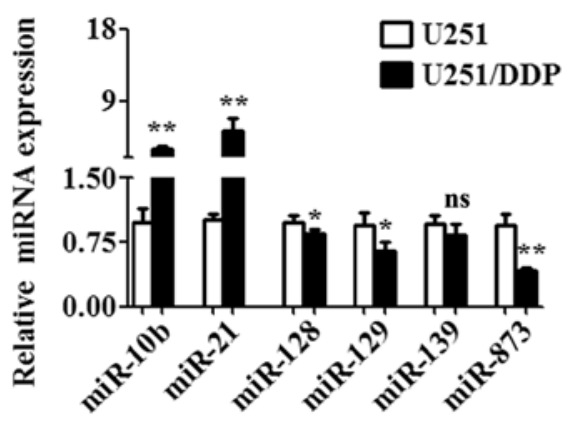

$\mathrm{C}$

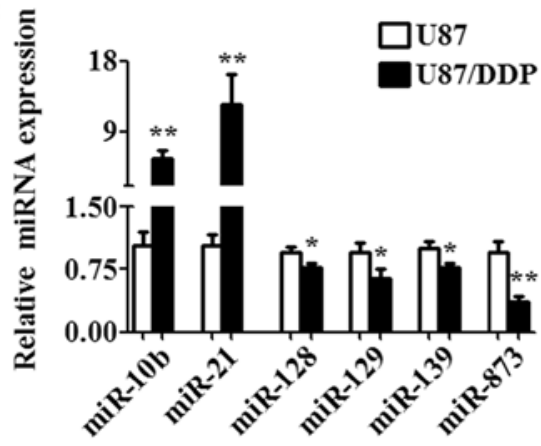

$\mathbf{F}$

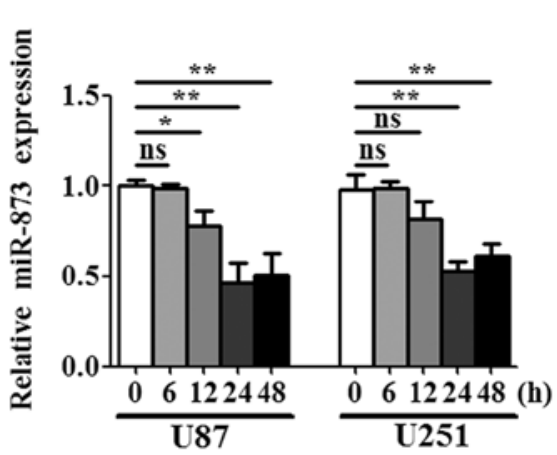

Figure 1. miR-873 expression in glioma tissues and cells. (A) qRT-PCR detection of miR-873 in twelve high-grade glioma tissues and seven normal brain tissues. (B) The $\mathrm{IC}_{50}$ level of U87 and U87/DDP. (C) qPCR detection of differential miRNAs expression in U87 and U87/DDP cells. (D) The IC ${ }_{50}$ level of U251 and U251/DDP. (E) qPCR detection of differential miRNAs expression in U251 and U251/DDP cells. (F) The effect of cisplatin on the expression of miR-873. $\mathrm{U} 87$ and $\mathrm{U} 251$ cells were exposed to $\mathrm{IC}_{50}$ value of cisplatin for the indicated time. qPCR was used to determine miR-873 levels.

with those of seven normal brains by qPCR. The results showed that miR-873 was significantly downregulated in the high-grade glioma tissues compared with the normal tissues (Fig. 1A). In addition, differential expression of miRNA was frequently related to drug resistance in gliomas $(10,11,18)$. Therefore, we generated the cisplatin-resistant glioma cells designated U87/DDP and U251/DDP to determine the correlation of dysregulated miR-873 with cisplatin resistance. We first determined the $\mathrm{IC}_{50}$ for the wild-type cells and resistant cells. We found that the $\mathrm{IC}_{50}$ for U87/DDP (Fig. 1B) or U251/ DDP (Fig. 1D) was significantly higher than that in the respective wild-type cells (U87 or U251). Then, we found that several miRNAs that had been identified in the previous cluster analysis were dysregulated in the U87/DDP or U251/ DDP cells (Fig. 1C and E). Among these, miR-873 was significantly downregulated in U87/DDP or U251/DDP cells compared to the respective wild-type cells (Fig. 1C and E). Next, we assessed the effects of cisplatin on the expression of miR-873 in U87 and U251 cells. The expression of miR-873 was downregulated in a time-dependent manner after treatment with the $\mathrm{IC}_{50}$ value of cisplatin (U87 cells were treated with $6.47 \mu \mathrm{g} / \mathrm{ml}$ and $\mathrm{U} 251$ cells were treated with $1.89 \mu \mathrm{g} / \mathrm{ml}$ ) (Fig. 1F). These data suggested that miR-873 might influence the susceptibility of glioma cells to cisplatin.

Overexpression of miR-873 attenuated the tumorigenic properties of cisplatin-resistant glioma cells. To assess the function of miR-873 on cisplatin-resistant glioma cells, $50 \mathrm{nM}$ miR-873 mimics or $50 \mathrm{nM}$ negative control (NC) mimics, which was used as a control, was transfected into U87/DDP and U251/DDP cells. Forty-eight hours after the transfection, the $\mathrm{qPCR}$ results indicated that $\mathrm{miR}-873$ expression was significantly upregulated in the miR-873 mimics transfected cells compared to the NC mimics transfected cells (Fig. 2A). Then, we examined cell proliferation by MTT assay for indicated time and the results showed that miR-873 significantly inhibited cell proliferation at 48 and $72 \mathrm{~h}$ (Fig. 2B and C). The colony formation assay gave the same conclusion as the MTT assay in the U87/DDP and U251/DDP cells (Fig. 2D). Moreover, we tested the effects of exogenous miR-873 expression on cell migration and invasion. Overexpression of miR-873 decreased both the migration (Fig. 2E) and invasiveness (Fig. 2F) of U87/DDP and U251/DDP cells as determined by Transwell assay. Furthermore, miR- 873 obviously incresed apoptosis of U87/DDP and U251/DDP cells as determined by caspase-3/7 activity analysis (Fig. 2G). Collectively, these findings indicated that miR-873 acts as a tumor suppressor and might relate to cisplatin resistance of glioma cells.

Overexpression of miR-873 sensitized glioma cells to cisplatin. To further investigate the function of miR- 873 on the cisplatin resistance, the miR-873 or NC mimics was transfected into U87/DDP and U87 cells. At first, we performed the MTT and colony formation assays to determine the effect of miR-873 on the proliferation of glioma cells. After being transfected with the miR-873 or NC mimics for $24 \mathrm{~h}$, the glioma cells were exposed to cisplatin for another 24-72 h. 
A

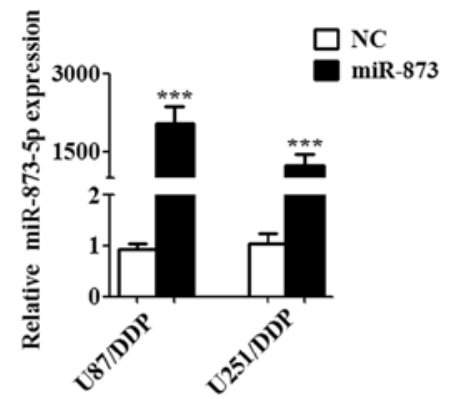

D

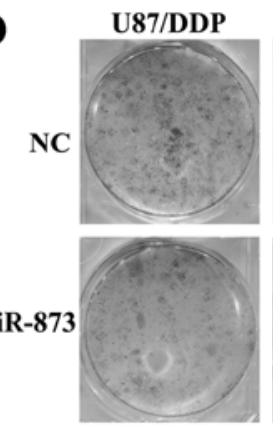

$\mathbf{F}$

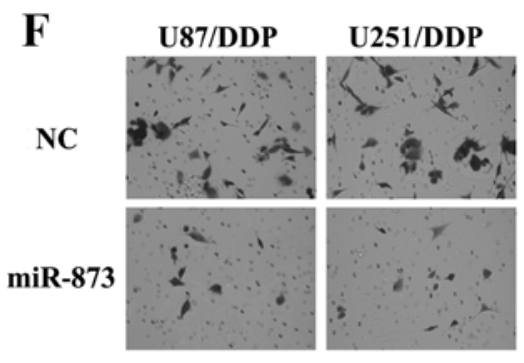

U251/DDP
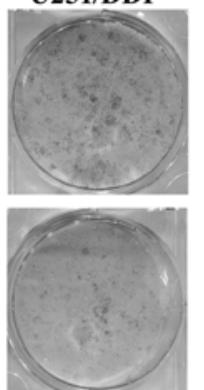

B

$\mathbf{E}$
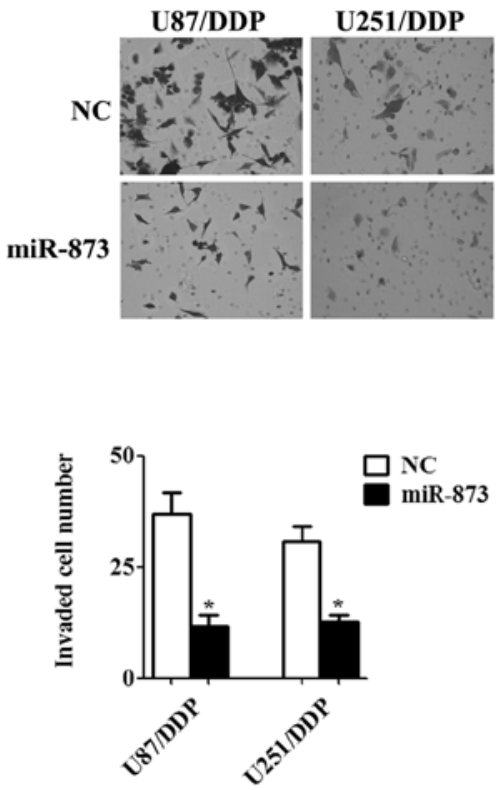

C

U87/DDP

$\rightarrow \mathrm{NC}$
$\rightarrow$ miR-873

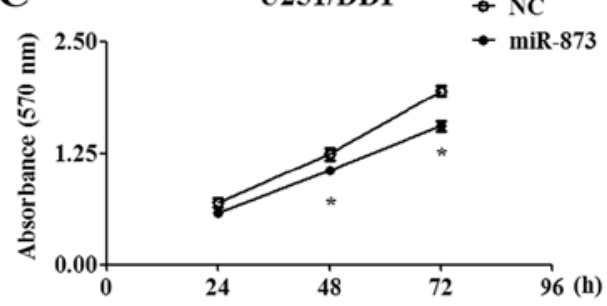

$\mathbf{G}$

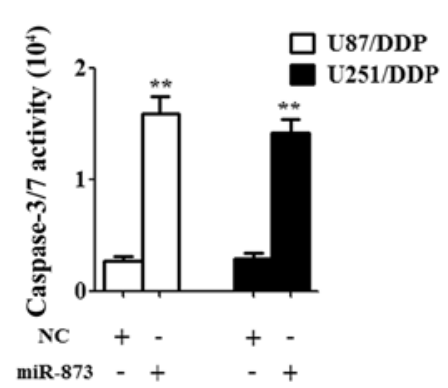

Figure 2. miR-873 decreased the tumorigenic properties of cisplatin-resistant glioma cells. (A) The related expression of miR-873 in cisplatin-resistant glioma cells was determined by qPCR after transfection with the miR-873 or NC mimics for $48 \mathrm{~h}$. (B) Cell proliferation was assessed for the miR-873 or NC mimics transfected U87/DDP cells by MTT assay. (C) Cell proliferation was assessed for the miR-873 or NC mimics transfected U251/DDP cells by MTT assay. (D) U87/DDP and U251/DDP cells were transfected with the miR-873 or NC mimics. As indicated, the cells were plated in triplicate for 2 weeks. (E) The effect of miR-873 on U87/DDP and U251/DDP cell migration was examined by Transwell assay after transfected with the miR-873 or NC mimics for $12 \mathrm{~h}$. A representative field of cells was available at a magnification of $x 200 .{ }^{* *} \mathrm{P}<0.01$. (F) The effect of miR-873 on U87/DDP and U251/DDP cell invasion was examined by Transwell assay after transfected with the miR-873 or NC mimics for $24 \mathrm{~h}$. ${ }^{*} \mathrm{P}<0.05$. (G) miR-873 increased caspase-3/7 activity in both U87/DDP and U251/DDP cells. The caspase-3/7 activity was assayed using a caspase-3/7 activity assay kit.

Then, the absorbance of glioma cells was measured using a microplate reader at $570 \mathrm{nM}$. The results of the MTT assay showed that miR-873 could enhance the anti-proliferative effect of cisplatin in both the cisplatin-resistant and wild-type glioma cells (Fig. 3A and B). The colony formation assay gave the same results as MTT assay in the U87/DDP and U87 cells (Fig. 3C). Later, we used flow cytometry to analyze the effects of miR-873 on cisplatin-induced apoptosis of the U87/DDP and U87 cells. These results also showed that miR-873 was able to significantly increase cisplatin-induced apoptosis of the U87/DDP and U87 cells after $48 \mathrm{~h}$ of cisplatin treatment (Fig. 3D). Moreover, western blotting showed a decreased expression of Bcl-2 and an increased expression of cleaved PARP, while the caspase-3/7 activity assay showed an increased activity of caspase-3/7 in the miR-873 mimic-transfected U87/DDP and U87 cells when exposed to cisplatin treatment (Fig. 3E and F). Thus, overexpression of miR-873 could decrease the resistance of the glioma cells to cisplatin.
$B c l-2$ is a functional target of miR-873. It is known that miRNAs perform their functions by regulating the expression of their target genes (19). Thus, the targets of miR-873 were predicted by miRanda and TargetScan, two publicly available prediction tools. We selected several putative targets that were related to cisplatin resistance from the predictions. The 3'UTR containing the potential binding site were cloned into pMiR-Reporter vector and luciferase assays were performed by transfection into U87/DDP cells. After co-transfection with 3'UTRs and the miR-873 or NC mimics for $24 \mathrm{~h}$, luciferase assays showed that the activity of Bcl-2 3'UTR was strongest inhibited by miR-873 (Fig. 4A). Furthermore, we cloned a mutated 3'UTR into the pMiR-Reporter vector to create a pMiR-Bcl-2-mu vector (Fig. 4B). After co-transfection of the U87/DDP cells with the miR-873 or NC mimics and the pMiR-Bcl-2-wt vector or pMiR-Bcl-2-mu vector for $24 \mathrm{~h}$, a luciferase assay was performed. The results showed that the luciferase activity was decreased by transfection with the miR-873 mimic (Fig. 4C). However, no significant difference 

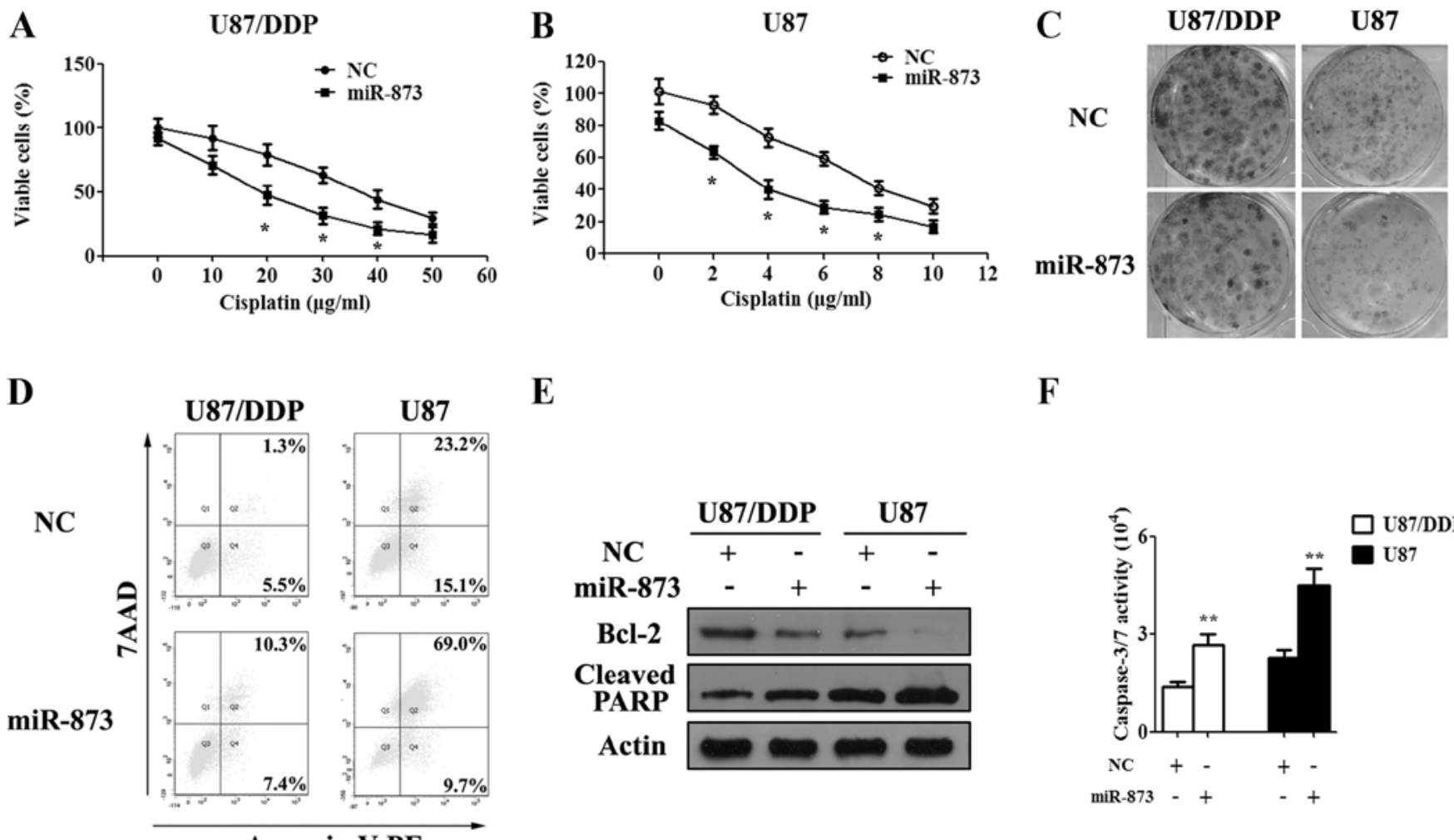

E

F
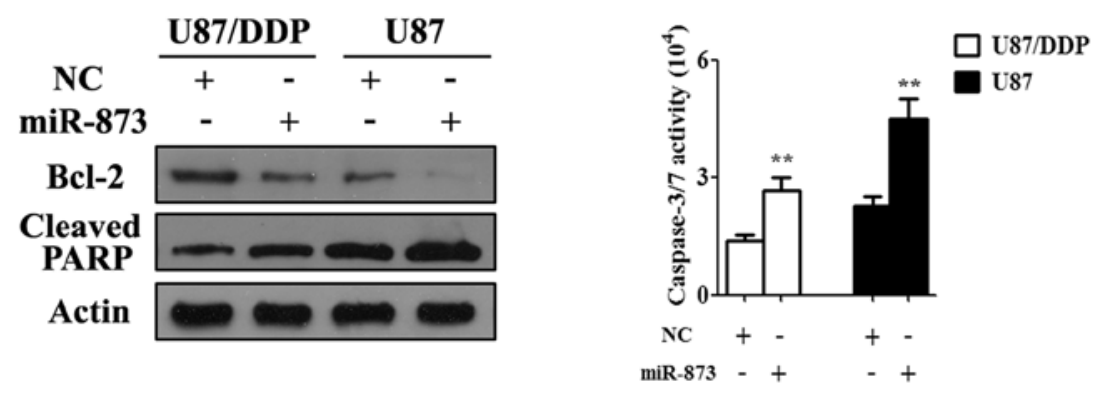

Figure 3. The effect of miR-873 on sensitivity of glioma cells to cisplatin. (A) After transfection with the miR-873 or NC mimics for 48 h, dose response curves of cisplatin in U87/DDP cells were measured by MTT assay. (B) After transfection with the miR-873 or NC mimics for 48 h, dose response curves of cisplatin in U87 cells were measured by MTT assay. (C) U87/DDP and U87 cells were transfected with the miR-873 or NC mimics. As indicated, the cells were plated in triplicate and exposed to the $\mathrm{IC}_{50}$ value of cisplatin for $48 \mathrm{~h}$. Then the cells were washed with PBS and the cell medium were changed to fresh $10 \%$ fetal bovine serum (FBS) and cultured for another 2 weeks. (D) After transfection with the miR-873 or NC mimics for 24 h, the cisplatin-resistant and wild-type U87 cells were treated with cisplatin for another $48 \mathrm{~h}$. The cellular apoptosis rate was determined using flow cytometry. (E) The expression of Bcl-2 and cleaved PARP in cisplatin-resistant and wild-type U87 cells was assayed by western blotting. $\beta$-actin was assayed as a loading control. (F) The caspase-3/7 activity was assayed using a caspase-3/7 activity assay kit.

was found when the cells were transfected with the miR-873 or NC mimics combined with the pMiR-Bcl-2-mu vector (Fig. 4C). Subsequently, western blotting was performed to analyze the effect of miR-873 on $\mathrm{Bcl}-2$ protein expression. The results showed that Bcl-2 expression was significantly lower in the cells transfected with the miR-873 mimics for $72 \mathrm{~h}$ compared with the NC mimics both in U87/DDP and U251/DDP cells (Fig. 4D and E). Furthermore, we examined the Bcl-2 levels in twelve high-grade glioma patient tissues and those of seven normal brains tissues by western blotting. The results showed that $\mathrm{Bcl}-2$ was upregulated in the highgrade glioma tissues (Fig. 4F and G). There was an obvious negative correlation between these signals $(r=-0.731, \mathrm{P}<0.01$; Fig. 4H). Therefore, miR-873 could downregulate the expression of Bcl-2 by directly targeting the Bcl-2 3'UTR.

Re-expression of Bcl-2 attenuates the effect of miR-873 on cisplatin-resistant cells. To examine whether Bcl-2 expression mediates the phenotype associated with increased miR-873 expression, the CMV-MCS/Bcl-2 vector or the CMV-MCS empty vector as a control was transfected into U87/DDP cells. A western blot assay confirmed that the expression of the Bcl-2 protein was significantly upregulated in the U87/DDP cells (Fig. 5A). The MTT assay showed that re-expression of $\mathrm{Bcl}-2$ decreased the antiproliferation effect of miR-873 on U87/DDP cells at 48 and $72 \mathrm{~h}$ (Fig. 5B). To further investigate the antitumor effect of miR-873 on U87/ DDP cells, Transwell assay and caspase-3/7 activity analysis were performed. Consistent with MTT result, re-expression of $\mathrm{Bcl}-2$ recovered the migration (Fig. $5 \mathrm{C}$ and $\mathrm{D}$ ) and invasive (Fig. 5C and E) ability of U87/DDP cells and abrogated the pro-apoptotic effect of miR-873 (Fig. 5F). These data further suggested Bcl-2 might be a functional target of miR- 873 .

Re-expression of $\mathrm{Bcl}-2$ decreases the effect of $\mathrm{miR}-873$ on cisplatin sensitivity. Having demonstrated that $\mathrm{Bcl}-2$ is a direct target of miR-873, we further investigated the role of Bcl-2 in cisplatin sensitivity of miR-873. After being co-transfected with the miR-873 or NC mimics and the CMV-MCS/Bcl-2 or CMV-MCS vector for $24 \mathrm{~h}$, the U87/ DDP cells were treated with $\mathrm{IC}_{50}$ value of cisplatin for another 24-72 h. The MTT assay showed that the proliferation was inhibited in the miR-873 mimic-transfected cells compared to the NC mimic-transfected cells at 48 and $72 \mathrm{~h}(\mathrm{P}<0.01$; Fig. 6A). However, co-transfection with the miR-873 mimics and $\mathrm{CMV}-\mathrm{MCS} / \mathrm{Bcl}-2$ vectors decreased the miR-873induced sensitivity of the U87/DDP cells to cisplatin (Fig. 6A). The colony formation assay gave the same result as the MTT assay in the U87/DDP cells (Fig. 6B). Furthermore, we determined whether overexpression of Bcl-2 could impair 


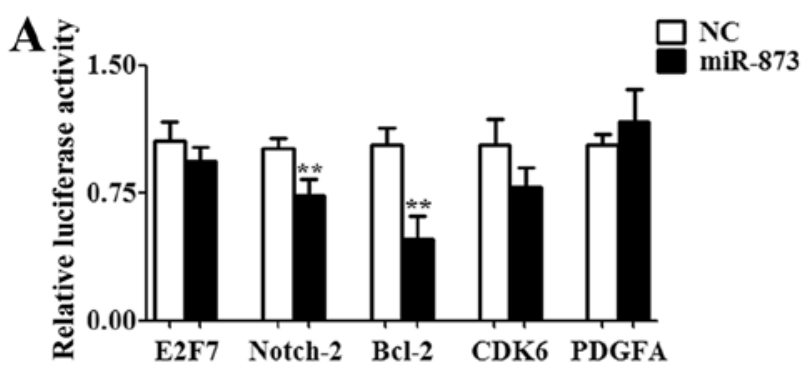

C

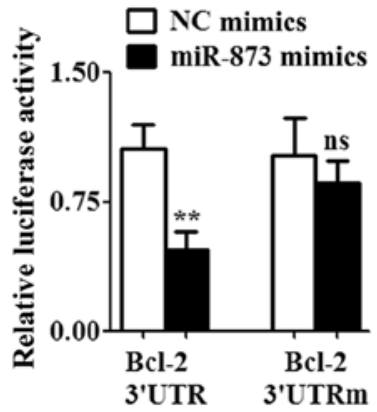

F

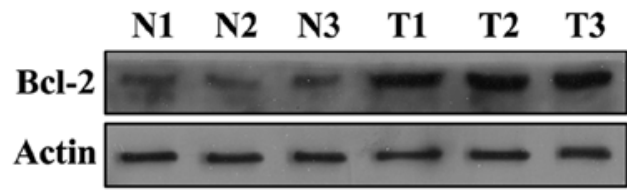

D

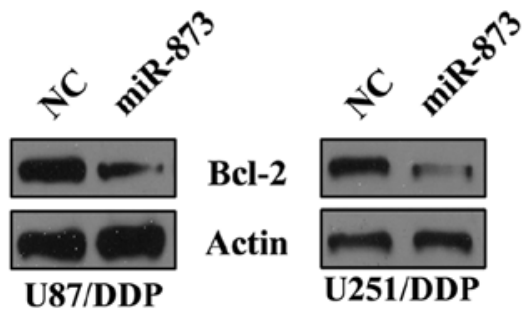

G

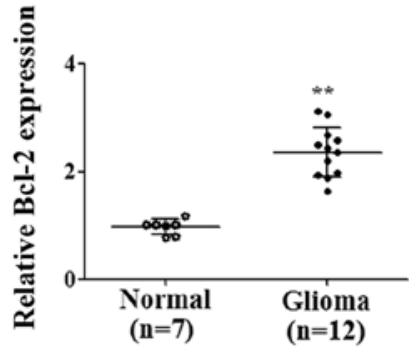

B

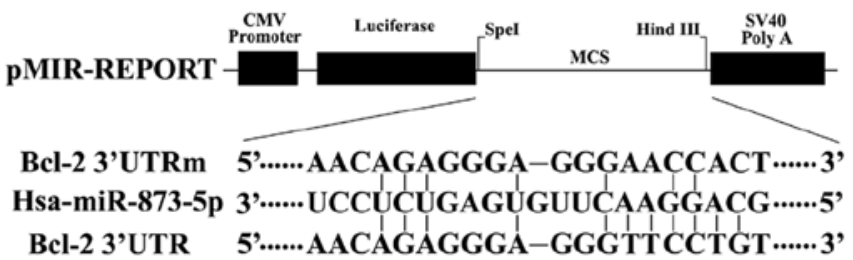

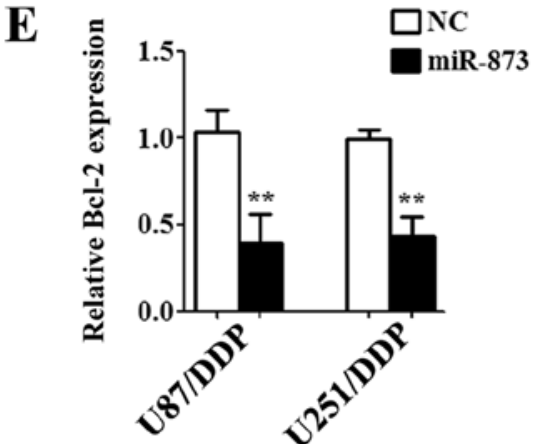

H

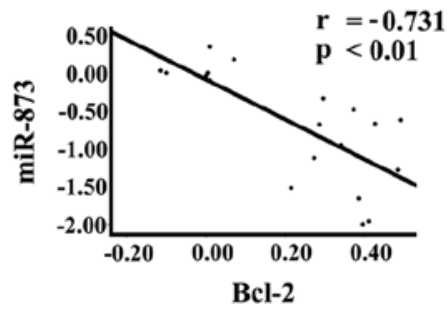

Figure 4. Bcl-2 is a direct target of miR-873 in glioma cells. (A) Luciferase assays were performed for U87/DDP cells cotransfected with the miR-873 or NC mimics together with control pMiR-Reporter vector or with pMiR-Reporter vector inserted sequence of the predicted 3'UTRs from the E2F7, Notch-2, Bcl-2, CDK6 or PDGFA genes. (B) Diagram of the seed sequence of miR-873 matched the 3'UTR of Bcl-2 (Bcl-2-3'UTR) and positions of the mutated nucleotides in the 3'UTR of Bcl-2 (Bcl-2-3'UTRm). (C) Relative luciferase activity was analyzed after co-transfection of wild-type or mutant 3'UTR reporter vector and $50 \mathrm{nM}$ miR-873 or NC mimics in U87/DDP cells for $24 \mathrm{~h}$. The data show the means \pm SD of the normalized luciferase activity of three independent experiments. (D) Western blot detection of Bcl-2 protein after transfection with the miR-873 or NC mimics for $72 \mathrm{~h}$ in U87/DDP and U251/DDP cells. $\beta$-actin was assayed as a loading control. (E) ImageJ densitometric analysis of Bcl-2 expression from immunoblots. The data are expressed as the means \pm SD of three independent experiments. (F) Western blot assays of Bcl-2 in the high-grade glioma tissues and normal brain tissues. (G) The 12 high-grade glioma samples were assessed for Bcl-2 expression by western blotting. Expression is shown for normal brain (normal, $\mathrm{n}=7$ ) samples and high-grade glioma (glioma, $\mathrm{n}=12$ ) samples. ${ }^{* *} \mathrm{P}<0.01$. (H) Bcl-2 was inversely correlated with miR-873 expression in high-grade glioma tissues.

the effect of miR-873 on sensitivity to cisplatin using flow cytometry and the caspase-3/7 activity assay. In the U87/ DDP cells, decreases in the caspase-3/7 activity and apoptosis rate were observed in cells co-transfected with the miR-873 mimics and the CMV-MCS/Bcl-2 vector cell compared to co-transfected with the miR-873 mimics and the CMV-MCS vector after treatment with cisplatin for $48 \mathrm{~h}$ (Fig. 6C and D). Taken together, these results indicated that re-expression of Bcl-2 was able to reduce the sensitivity-enhancing effect of miR-873 to cisplatin.

\section{Discussion}

Resistance to chemotherapeutic agents is a vital challenge in the treatment of glioma patients. In this study, we investigated the function of miR-873 on the cisplatin resistance of glioma cells. To our knowledge, this is the first report that miR-873 significantly decreased tumor chemoresistance, as measured by MTT assay, colony formation assay, flow cytometry, western blotting and caspase-3/7 activity assay. Furthermore, we demonstrated for the first time that miR-873 enhanced the sensitivity of cisplatin by targeting the mRNA 3'UTR of Bcl-2 and that miR-873 and Bcl-2 were inversely dysregulated in glioma. Thus, these results indicated that miR-873 could serve as a molecular target for regulating the glioma cell sensitivity to cisplatin.

Previous studies have shown that aberrant miRNA expression was implicated in the progression of chemoresistance in gliomas. Guo et al demonstrated that Let-7b was significantly decreased in cisplatin-resistant glioma cells compared to wild-type cells, and overexpression of Let-7b significantly increased cisplatin-induced apoptosis (10). Moreover, it has been reported that miR-106a was significantly upregulated in cisplatin-resistant and gefitinib-resistant glioma cells and that knockdown of miR-106a enhanced the temozolomideinduced apoptosis in glioma cells (11). Furthermore, it has 

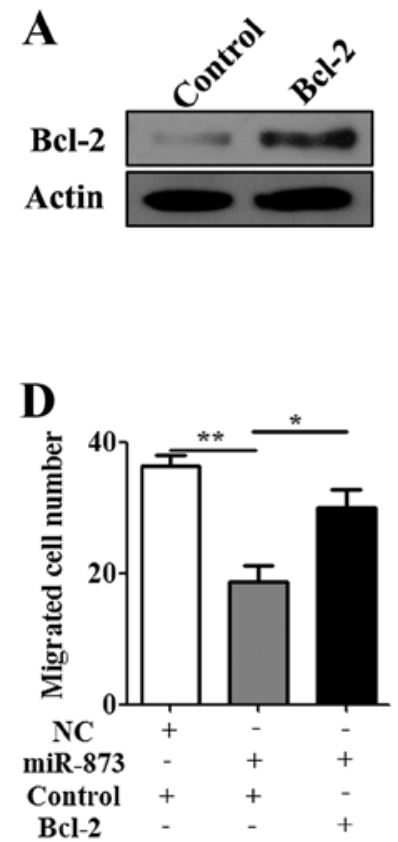
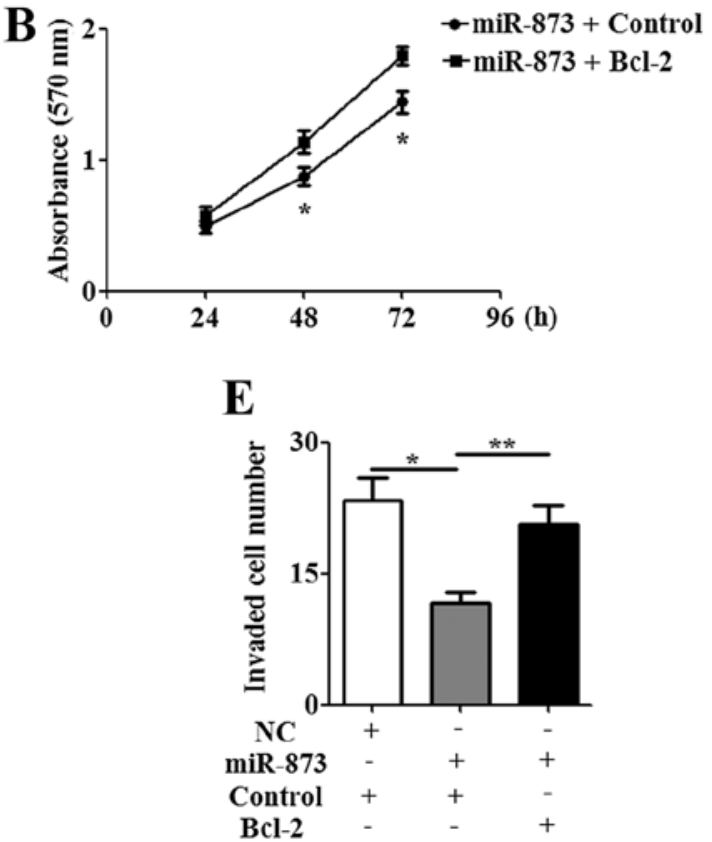

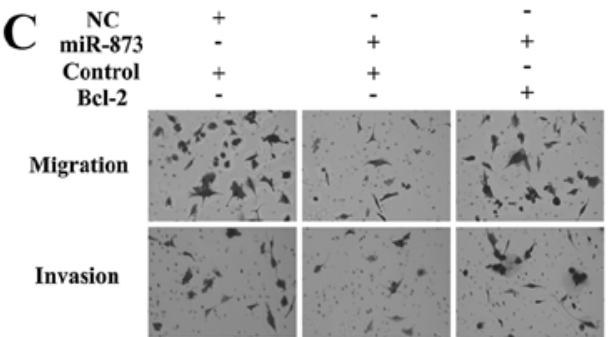

$\mathbf{F}$

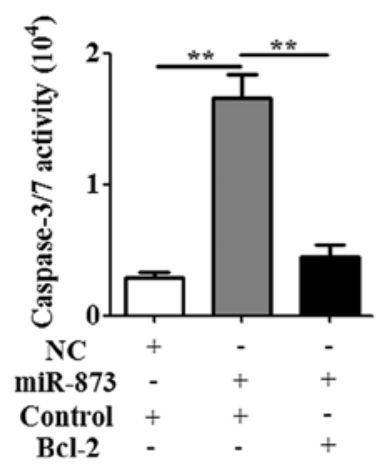

Figure 5. Ectopic expression of Bcl-2 partially abrogates the effect of miR-873. (A) Western blot detection of Bcl-2 protein after transfection with CMV-MCS/ Bcl-2 vector or CMV-MCS empty vector for $72 \mathrm{~h}$ in U87/DDP cells. $\beta$-actin was assayed as a loading control. (B) Cell proliferation was assessed for miR-873 mimics and the CMV-MCS/Bcl-2 or CMV-MCS vector transfected U87/DDP cells by MTT assay. (C) The effects of miR-873 on U87/DDP cell migration and invasion were examined by Transwell assay after transfected with the miR-873 or NC mimics and the CMV-MCS/Bcl-2 or CMV-MCS vector. (D and E) A representative field of cells was available at a magnification of $\mathrm{x} 200 .{ }^{*} \mathrm{P}<0.05,{ }^{* *} \mathrm{P}<0.01$. (F) The caspase-3/7 activity was assayed using a caspase-3/7 activity assay kit.

A

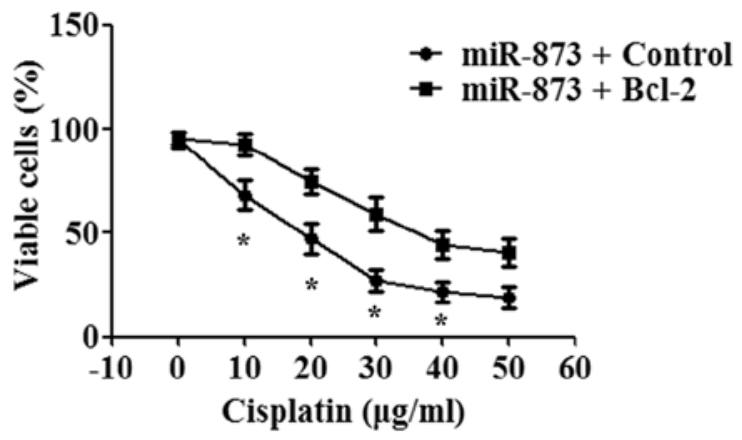

C
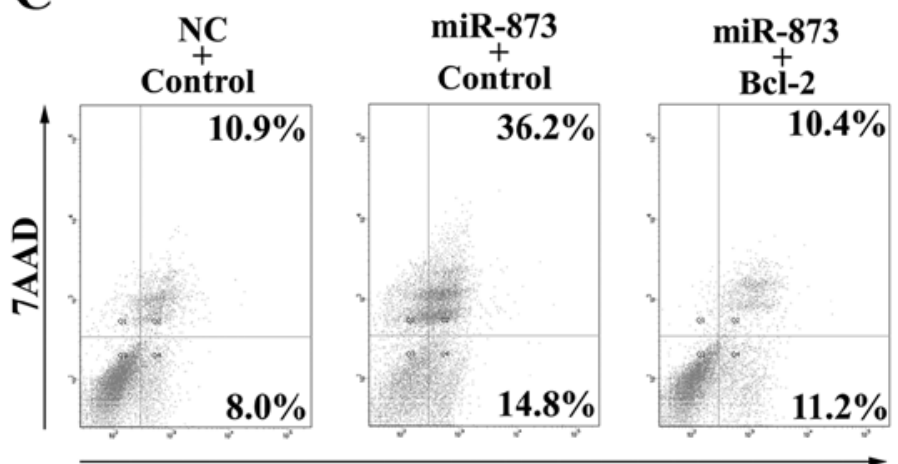

Annexin V-PE

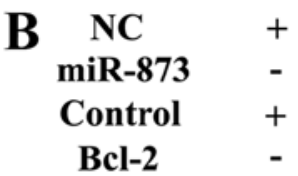

D

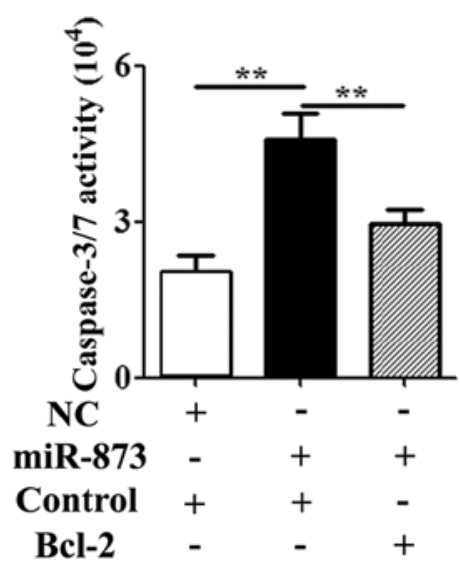

Figure 6. Enforced expression of Bcl-2 decreased the effect of miR-873 on cisplatin sensitivity. (A) After transfection with the miR-873 mimics and the CMV-MCS/Bcl-2 or CMV-MCS vector for $48 \mathrm{~h}$, dose response curves of cisplatin in U87/DDP cells were measured by MTT assay. (B) U87/DDP cells were co-transfected with the miR-873 or NC mimics and the CMV-MCS/Bcl-2 or CMV-MCS vector. As indicated, the cells were plated in triplicate and exposed to the $\mathrm{IC}_{50}$ value of cisplatin for $48 \mathrm{~h}$. Then the cells were washed with PBS and the cell medium were changed to fresh $10 \%$ fetal bovine serum (FBS) and cultured for another 2 weeks. (C) After co-transfection with the miR-873 or NC mimics and the CMV-MCS/Bcl-2 or CMV-MCS vector for $24 \mathrm{~h}$, the U87/ DDP cells were treated with cisplatin for another $48 \mathrm{~h}$. The cell apoptosis rate was determined using flow cytometry. (D) The caspase-3/7 activity was assayed using a caspase- $3 / 7$ activity assay kit. 
been shown that miR-136 and miR-139 were downregulated in human glioma tissues and cells, and overexpression of miR-136 or miR-139 promotes cisplatin-induced or temozolomide-induced apoptosis in glioma $(12,13,18)$. Based on these observations, dysregulated expression of miRNA may determine the response of glioma cells to chemotherapy. In this study, we found that miR-873 was downregulated in high-grade glioma tissues compared to normal tissues and deceased in cisplatin-resistant glioma cells compared with wild-type cells. These results indicated that miR-873 may affect the sensitivity of glioma cells to cisplatin. However, the cause of the low expression of miR-873 in the cisplatinresistant glioma cells remains to be determined in further studies.

To date, few studies have reported on the function of miR-873. Liu et al demonstrated that when stimulated by IL-17, miR-873 promotes the activation of NF- $\kappa B$ and the production of inflammatory cytokines in human multiple sclerosis (16). It has also been reported that miR-873 is involved in the regulation of the Hedgehog signaling and has relevance to physiological cranial bone development (20). miR-873 is downregulated in tamoxifen-resistant breast tumor cell lines, while overexpression of $\mathrm{miR}-873$ reverses the tamoxifen resistance by targeting cyclin-dependent kinase 3 (CDK3) (15). More importantly, cluster analysis revealed that miR-873 is significantly downregulated in glioblastomas (17) and that ectopic expression of miR-873 reduces cell proliferation, migration and invasion of glioma cells by targeting IGF2BP1 (14). Here, we demonstrated that overexpression of miR-873 sensitized glioma cells to cisplatin by arresting cell proliferation and inducing apoptosis as indicated by the results of the MTT assay, colony formation assay, flow cytometry, western blotting and caspase-3/7 activity assay. However, whether miR-873 could sensitize glioma cells to other chemotherapeutic drugs remains to be determined.

Bcl-2, a key anti-apoptotic protein, functions as an oncogene on the basis that it promotes migration and invasiveness of glioma cells $(21,22)$. Moreover, inhibition of Bcl-2 expression enhances the chemosensitivity of glioma cells, including sensitivity to cisplatin (23). Many studies have confirmed that $\mathrm{Bcl}-2$ is an important target for miRNA in the regulation of the chemosensitivity of glioma cells $(13,24,25)$. In this study, we used the luciferase assay to demonstrate that Bcl-2 was a functional target of miR-873. Ectopic expression of Bcl-2 could offset the miR-873-induced sensitivity of the glioma cells to cisplatin. Nevertheless, many more genes are targeted by miR-873, and the additional target genes need to be explored further.

In conclusion, we demonstrated that miR-873 was downregulated in cisplatin-resistant glioma cells compared to wild-type cells and that miR-873 enhanced the sensitization to cisplatin by targeting Bcl-2. Our data suggest that miR-873 might be a potential biomarker and a promising therapeutic strategy for cisplatin-resistant glioma cells.

\section{Acknowledgements}

This study was supported by the National Natural Sciences Foundation of China (nos. 81171127, 81371422, 81171577 and 81371790).

\section{References}

1. Baraniskin A, Kuhnhenn J, Schlegel U, Maghnouj A, Zöllner H, Schmiegel W, Hahn S and Schroers R: Identification of microRNAs in the cerebrospinal fluid as biomarker for the diagnosis of glioma. Neuro-oncol 14: 29-33, 2012.

2. Gabayan AJ, Green SB, Sanan A, Jenrette J, Schultz C, Papagikos M, Tatter SP, Patel A, Amin P, Lustig R, et al: GliaSite brachytherapy for treatment of recurrent malignant gliomas: A retrospective multi-institutional analysis. Neurosurgery 58: 701-709, discussion 701-709, 2006

3. Rocha CR, Garcia CC, Vieira DB, Quinet A, de AndradeLima LC, Munford V, Belizário JE and Menck CF: Glutathione depletion sensitizes cisplatin- and temozolomide-resistant glioma cells in vitro and in vivo. Cell Death Dis 6:e1727, 2015.

4. Cai Y, Yu X, Hu S and Yu J: A brief review on the mechanisms of miRNA regulation. Genomics Proteomics Bioinformatics 7: 147-154, 2009.

5. Patnaik S, Mallick R, Kannisto E, Sharma R, Bshara W, Yendamuri S and Dhillon SS: MiR-205 and MiR-375 microRNA assays to distinguish squamous cell carcinoma from adenocarcinoma in lung cancer biopsies. J Thorac Oncol 10: 446-453, 2015.

6. Khan S, Wall D, Curran C, Newell J, Kerin MJ and Dwyer RM: MicroRNA-10a is reduced in breast cancer and regulated in part through retinoic acid. BMC Cancer 15: 345, 2015.

7. Liu K, Zhang C, Li T, Ding Y, Tu T, Zhou F, Qi W, Chen H and Sun X: Let-7a inhibits growth and migration of breast cancer cells by targeting HMGA1. Int J Oncol 46: 2526-2534, 2015.

8. Wang YW, Chen X, Gao JW, Zhang H, Ma RR, Gao ZH and Gao P: High expression of cAMP-responsive element-binding protein 1 (CREB1) is associated with metastasis, tumor stage and poor outcome in gastric cancer. Oncotarget 6: 10646-10657, 2015.

9. Lai NS, Wu DG, Fang XG, Lin YC, Chen SS, Li ZB and Xu SS: Serum microRNA-210 as a potential noninvasive biomarker for the diagnosis and prognosis of glioma. Br J Cancer 112 (Suppl): 1241-1246, 2015.

10. Guo Y, Yan K, Fang J, Qu Q, Zhou M and Chen F: Let-7b expression determines response to chemotherapy through the regulation of cyclin D1 in glioblastoma. J Exp Clin Cancer Res 32: 41, 2013.

11. Wang Q, Wang Z, Chu L, Li X, Kan P, Xin X, Zhu Y and Yang P: The effects and molecular mechanisms of miR-106a in multidrug resistance reversal in human glioma U87/DDP and U251/G cell lines. PLoS One 10: e0125473, 2015.

12. Chen W, Yang Y, Chen B, Lu P, Zhan L, Yu Q, Cao K and Li Q: MiR-136 targets E2F1 to reverse cisplatin chemosensitivity in glioma cells. J Neurooncol 120: 43-53, 2014.

13. Yang Y, Wu J, Guan H, Cai J, Fang L, Li J and Li M: MiR-136 promotes apoptosis of glioma cells by targeting AEG-1 and Bcl-2. FEBS Lett 586: 3608-3612, 2012.

14. Wang RJ, Li JW, Bao BH, Wu HC, Du ZH, Su JL, Zhang MH and Liang HQ: MicroRNA-873 (miRNA-873) inhibits glioblastoma tumorigenesis and metastasis by suppressing the expression of IGF2BP1. J Biol Chem 290: 8938-8948, 2015.

15. Cui J, Bi M, Overstreet AM, Yang Y, Li H, Leng Y, Qian K, Huang Q, Zhang C, Lu Z, et al: MiR-873 regulates Era transcriptional activity and tamoxifen resistance via targeting CDK3 in breast cancer cells. Oncogene: 22, Dec 2014 (Epub ahead of print). doi:10.1038/onc.2014.430.

16. Liu X, He F, Pang R, Zhao D, Qiu W, Shan K, Zhang J, Lu Y, Li Y and Wang Y: Interleukin-17 (IL-17)-induced microRNA 873 (miR-873) contributes to the pathogenesis of experimental autoimmune encephalomyelitis by targeting A20 ubiquitinediting enzyme. J Biol Chem 289: 28971-28986, 2014.

17. Skalsky RL and Cullen BR: Reduced expression of brain-enriched microRNAs in glioblastomas permits targeted regulation of a cell death gene. PLoS One 6: e24248, 2011.

18. Li RY, Chen LC, Zhang HY, Du WZ, Feng Y, Wang HB, Wen JQ, Liu X, Li XF, Sun Y, et al: MiR-139 inhibits Mcl-1 expression and potentiates TMZ-induced apoptosis in glioma. CNS Neurosci Ther 19: 477-483, 2013.

19. Lynam-Lennon N, Maher SG and Reynolds JV: The roles of microRNA in cancer and apoptosis. Biol Rev Camb Philos Soc 84: 55-71, 2009.

20. Koufaris C, Papagregoriou G, Kousoulidou L, Moutafi M, Tauber M, Jouret B, Kieffer I, Deltas C, Tanteles GA, Anastasiadou V, et al: Haploinsufficiency of the miR-873/ miR-876 microRNA cluster is associated with craniofacial abnormalities. Gene 561: 95-100, 2015. 
21. Wick W, Wagner S, Kerkau S, Dichgans J, Tonn JC and Weller M: BCL-2 promotes migration and invasiveness of human glioma cells. FEBS Lett 440: 419-424, 1998.

22. Wick W, Wild-Bode C, Frank B and Weller M: BCL-2-induced glioma cell invasiveness depends on furin-like proteases. J Neurochem 91: 1275-1283, 2004.

23. Zhu CJ, Li YB and Wong MC: Expression of antisense bcl-2 cDNA abolishes tumorigenicity and enhances chemosensitivity of human malignant glioma cells. J Neurosci Res 74: 60-66, 2003
24. Wang F, Li T, Zhang B, Li H, Wu Q, Yang L, Nie Y, Wu K, Shi Y and Fan D: MicroRNA-19a/b regulates multidrug resistance in human gastric cancer cells by targeting PTEN. Biochem Biophys Res Commun 434: 688-694, 2013.

25. Zhu W, Xu H, Zhu D, Zhi H, Wang T, Wang J, Jiang B, Shu Y and Liu P: miR-200bc/429 cluster modulates multidrug resistance of human cancer cell lines by targeting BCL2 and XIAP. Cancer Chemother Pharmacol 69: 723-731, 2012. 